\title{
Bioluminescence-based ATP assays using a charge-coupled device imaging system
}

\author{
Robert D. Shepherd and Kristina D. Rinker \\ Colorado State University, Fort Collins, CO, USA
}

BioTechniques 37:208-210 (August 2004)

Knowing the viability of cell cultures before, during, and after experiments is an integral part of determining the nature of phenomena associated with work conducted in biomedical engineering laboratories. One of the best markers of viability is adenosine triphosphate (ATP), which is the primary energy source for all metabolic reactions and is present in all viable cells. As cellular processes are interrupted due to chemical effects, physical damage, or a depletion of essential nutrients or oxygen, ATP levels are diminished. This characteristic allows determination of released ATP to be a good measure of overall culture viability $(1,2)$. While several ATP assay methods exist, bioluminescent determination is of most interest from the standpoint of rapid screening of multiple samples. Bioluminescent measurement of ATP has been previously described $(1,3)$ and applied to automated high-throughput cytotoxicity screening in pharmaceutical research and development (4). While this level of sophistication and throughput is unnecessary at the bench level, the ease of use, speed, and accuracy of luminescent detection is attractive for viability determination in laboratories that employ differential chemical treatment to generate responses from cell cultures or tissue engineered constructs. This is the case in many biomedical engineering laboratories that are not traditional cell biology facilities. In these laboratories, it may be efficient to conduct viability experiments on more generalized equipment, such as a charge-coupled device (CCD) camera-based imaging system, which should be able to quantitate bioluminescent signals. Recently, a kit for the extraction and bioluminescent determination of ATP was made available. The ViaLight ${ }^{\mathrm{TM}}$ Plus kit (Cambrex,
Rockland, ME, USA) includes all of the reagents necessary to lyse cells and measure ATP in each well of a luminescence-compatible 96- or 384-well microplate. As designed, the assay requires reading of the plate via luminometer for determination of ATP concentration. The study presented here was designed to explore detection of ATP-dependent luminescence from the ViaLight Plus kit with a CCD-based BioChemi ${ }^{\mathrm{TM}}$ BioImaging ${ }^{\mathrm{TM}}$ System (UVP, Upland, CA, USA).

To test the ability to perform luminescence-based microplate viability experiments using an imaging system, it was decided to first conduct a feasibility analysis. A dilution profile based on a known number of live cells was employed to provide a detection limit and establish a decision point for moving forward with a more complicated and useful cytotoxicity assay based upon chemical exposure. All experiments were conducted with Mono Mac 6 (MM6) cells (DSMZ, Braunschweig, Germany), a human monocytic cell line, cultured in RPMI 1640 medium (Sigma, St. Louis, MO, USA) supplemented with $10 \%$ heat-inactivated bovine serum (Sigma) cultivated as described previously (5).

For dilution experiments, $1 \mathrm{~mL}$ of cell suspension was removed from an actively growing MM6 culture and used as a sample source for determination of cell density and viability via trypan blue exclusion. After establishing cell density and viability, a dilution study was conducted using a varying num- ber of cells added to a luminescencecompatible white 96-well microplate (Cambrex). The dilution range encompassed points at 70,000, 50,000, $40,000,25,000,12,500,6250$, and 0 live cells/well. The final volume of each well was $130 \mu \mathrm{L}$, prepared by combining appropriate volumes of cell suspension and phosphate-buffered saline (PBS) to achieve the desired number of live cells/well. Six replicates of each cell number were assayed according to ViaLight Plus Instructions (http://www.cambrex. com/Content/Documents/Bioscience/ ViaLight\%20Plus\%20Aw.pdf), and the resulting luminescence was recorded over a 7-min exposure at full aperture using the BioChemi BioImaging System. The captured image was analyzed using LabWorks ${ }^{\mathrm{TM}}$ software (UVP), resulting in an integrated optical density (IOD) signal value for each well. A Q-test was performed on the data, and the average IOD and standard deviation for each point was plotted. As shown in Figure 1, a linear relationship between average IOD and live cell number was obtained between 0 and 50,000 live cells/well.

To determine the applicability of the assay procedure on cells exposed to representative experimental conditions, a cytotoxicity study using staurosporine was undertaken. Staurosporine was chosen as an antagonist due to its ability to induce cell death via apoptosis in a wide variety of cell types $(6,7)$, including leukocytes. Nine staurosporine (Sigma) concentrations between 0.05 and $2.50 \mu \mathrm{M}$ were chosen

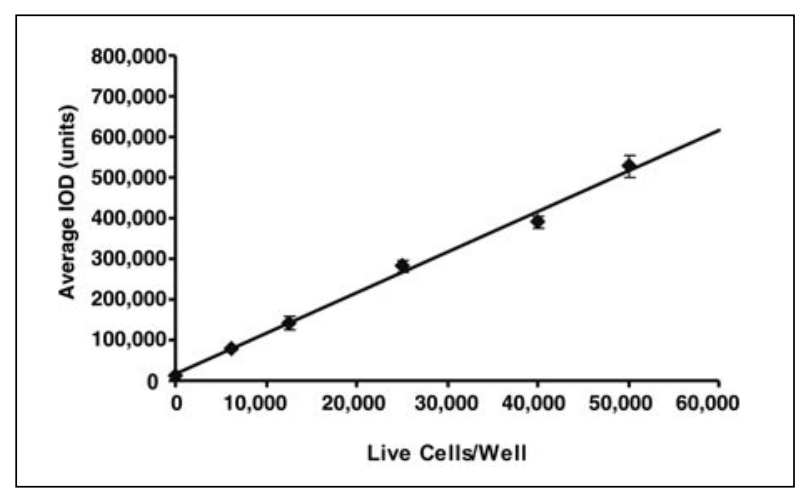

Figure 1. Dilution profile of live cells. Using the BioChemi System, the average integrated optical density (IOD) values were determined at the indicated numbers of live cells. Regression line equation: $\mathrm{y}=$ $10.005 \mathrm{x}+15.846, R^{2}=0.995$. Error bars represent standard deviation of replicate analyses. 


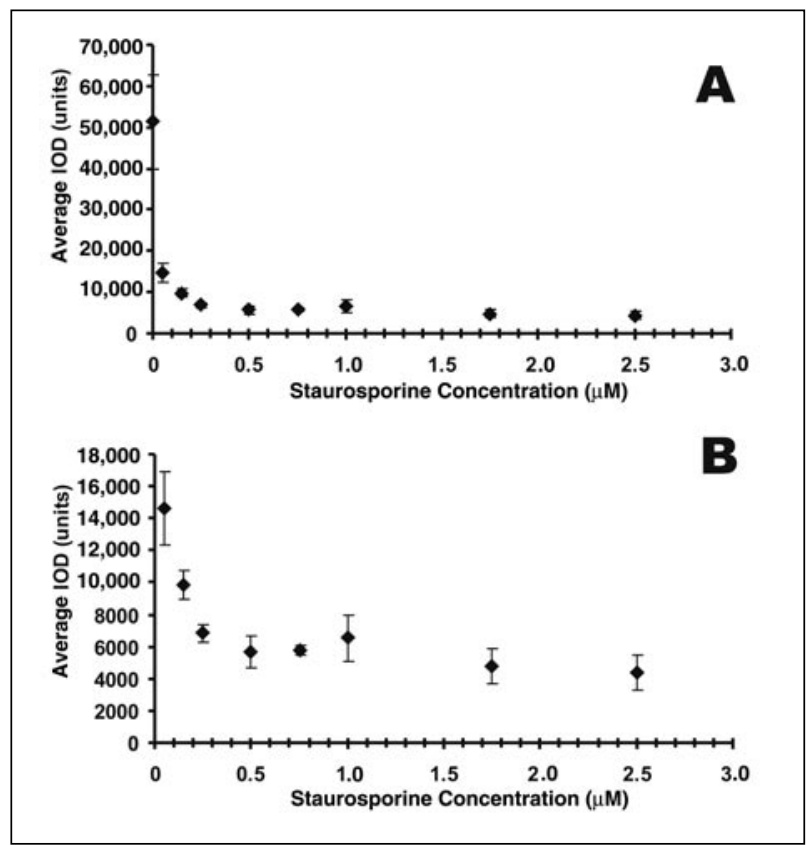

Figure 2. Effects of staurosporine on cytotoxicity. The BioChemi System was used to determine the average integrated optical density (IOD) for Mono Mac 6 cells treated with staurosporine. (A) Complete 9-point data set. (B) Data trend without zero point to allow discrimination of $0.05,0.15$, and $0.25 \mu \mathrm{M}$ points. Error bars represent standard deviation of replicate analyses.

and examined in duplicate. Eighteen flasks were seeded to an initial density of $3 \times 10^{5}$ live cells $/ \mathrm{mL}$ in fresh RPMI 1640 medium plus $10 \%$ bovine serum. The appropriate amount of staurosporine was then added to each flask. Cultures were left to incubate at $37^{\circ} \mathrm{C}$ and $5 \% \mathrm{CO}_{2}$ for $48 \mathrm{~h}$, at which time cell density and viability were determined by trypan blue exclusion. Based on these counts, a total of 35,000 cells/ well (no discrimination between live and dead) were plated as before in a 96-well luminescence-compatible microplate. Three replicates from each flask, a total of six samples for each treatment condition, were examined. The volume of each well was adjusted to $120 \mu \mathrm{L}$ with PBS, and the ViaLight Plus assay was conducted according to the instructions. Exposure time for these experiments was increased to $10 \mathrm{~min}$ at full aperture, and the resulting well signals were not as bright as those obtained from dilution experiments. Figure 2 demonstrates the results of the staurosporine experiments and indicates that the assay was able to discriminate changes in cell viability with staurosporine treatment.
Results of this study demonstrate the ability to use bioluminescent ATP assays as a rapid method for determining cell viability from large sample numbers with a CCDbased imaging system. The linear relationship obtained between live cell number/well and average IOD from 0 to 50,000 live cells/well showed the adequate sensitivity of the method for a wide range of culture assays. While experiments with fewer than 6250 live cells/ well were not undertaken in this study, it is likely that with assay optimization for specific conditions, significant differences in bioluminescence would be detectable at lower cell numbers yielding sensitivities approaching that of the ViaLight Plus assay performed on a luminometer. Staurosporine exposure results showed that the BioChemi System was able to discriminate between relatively small changes in chemical treatment conditions. While the working range of signal that was obtained was somewhat small $(0-0.25 \mu \mathrm{M})$, these studies were intended only to provide evidence of the feasibility of the technique. With optimization to address specific experimental requirements, sufficient sensitivity for a wide variety of cytotoxicity/ viability assays should be achievable. Based upon the presented results, the use of a CCD-based imaging system for bioluminescent ATP-based viability determination may provide a quick and reliable screening method applicable to a variety of biomedical engineering and bioscience laboratories.

\section{ACKNOWLEDGMENTS}

This work was partially supported by The Whitaker Foundation grant $R G$ 01-0145 and the National Institutes of
Health grant HL68916. The authors wish to thank Alex Batchelor of Cambrex Bio Science for the generous donation of ViaLight HS and ViaLight Plus kits and Lyndsey Carlaw for her assistance with cell culture and assay performance.

\section{COMPETING INTERESTS STATEMENT}

The authors declare that they have no competing interests.

\section{REFERENCES}

1.Crouch, S.P.M., R. Kozlowski, K.J. Slater, and J. Fletcher. 1993. The use of ATP bioluminescence as a measure of cell proliferation and cytotoxicity. J. Immunol. Methods 160:81-88

2.Dexter, S.J., M. Camara, M., Davies, and K.M. Shakesheff. 2003. Development of a bioluminescent ATP assay to quantify mammalian and bacterial cell number from a mixed population. Biomaterials 24:27-34.

3.Higashi, T., A. Isomoto, E. Tyuma, E. Kakishita, M. Uomoto, and K. Nagai. 1985. Quantitative and continuous analysis of ATP release from blood platelets with firefly luciferase luminescence. Thromb. Haemost. 53:65-69.

4.Slater, K. 2001. Cytotoxicity tests for highthroughput drug discovery. Curr. Opin. Biotech. 12:70-74.

5.Rinker, K.D., V. Prabakhar, and G.A. Truskey. 2001. Effect of contact time and force on monocyte adhesion to vascular endothelium. Biophys. J. 80:1722-1732.

6.Wertz, I.E. and M.R. Hanley. 1996. Diverse molecular provocation of programmed cell death. Trends Biochem. Sci. 21:359-364.

7.Belmokhtar, C.A., A. Torriglia, M.F. Counis, Y. Courtois, A. Jacquemin-Sablon, and E. Segal-Bendirdjian. 2000. Nuclear translocation of a leukocyte elastase inhibitor/elastase complex during staurosporineinduced apoptosis: role in the generation of nuclear L-DNase II activity. Exp. Cell Res. 254:99-109.

Received 25 February 2004; accepted 29 April 2004.

Address correspondence to Kristina D. Rinker, Colorado State University, Department of Chemical Engineering, $1370 \mathrm{Cam}$ pus Delivery, Fort Collins, CO 80523-1370, USA.e-mail: rinker@engr.colostate.edu 